\title{
Recursos e técnicas fisioterapêuticas que devem ser utilizadas com cautela ou evitadas em pacientes com COVID-19*
}

\author{
Physiotherapeutic techniques that should be used with caution or avoided in patients with \\ COVID-19*
}

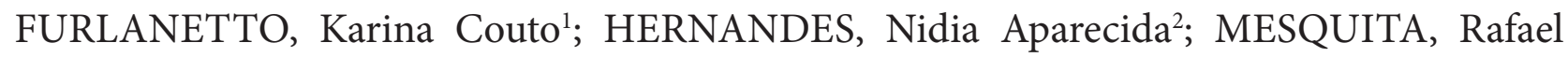
Barreto de ${ }^{3}$; em nome do Comitê COVID-19 da ASSOBRAFIR.

\begin{abstract}
Resumo
O presente documento apresenta direcionamentos para o atendimento de Fisioterapia a pacientes com suspeita ou diagnostico de COVID-19, com foco nos recursos e técnicas que devem ser utilizados com cautela ou até mesmo evitados. Em meio à pandemia da COVID-19, a redução da disseminação do vírus é uma preocupação importante dos órgãos e profissionais de saúde. Deste modo, recomendações relacionadas ao risco para a geração de gotículas e aerossóis, ao risco de causar gasto energético excessivo e ao risco de causar injúria pulmonar foram apresentadas. Recomenda-se utilizar com cautela ou evitar o uso de máscara de Venturi, tenda de oxigênio, cânula nasal de alto fluxo, ventilação não-invasiva com circuito único, ressuscitador manual, exercícios físicos com intensidade moderada-alta, assim como estratégias convencionais de ventilação mecânica. O fisioterapeuta que assiste pacientes com COVID-19 deve observar esses direcionamentos para garantir um atendimento eficaz e seguro para si próprio e para o paciente.
\end{abstract}

Palavras-chave: Fisioterapia; Padrão de Cuidado; COVID-19.

\footnotetext{
${ }^{*}$ Revisado por membros do Comitê COVID-19 da ASSOBRAFIR, nomeado por meio do memorando Nº03/2020. Esta publicação é uma atualização da Comunicação Oficial "Recursos e técnicas fisioterapêuticas que devem ser utilizadas com cautela ou evitadas em pacientes com COVID-19", chancelada pelo Comitê COVID-19 da ASSOBRAFIR, originalmente escrita pelos mesmos autores e divulgada em 16/04/2020 no endereço eletrônico https://assobrafir.com. br/covid-19-recursos-e-tecnicas-que-devem-ser-utilizadas-com-cautela-ou-evitadas/

${ }_{1}^{1}$ Programa de Pós-Graduação em Ciências da Reabilitação, Universidade Pitágoras-Unopar (UNOPAR), Londrina, Paraná, Brasil. Laboratório de Pesquisa em Fisioterapia Pulmonar (LFIP), Universidade Estadual de Londrina (UEL), Londrina, Paraná, Brasil. Email: ka furlanetto@hotmail.com. KCF - https://orcid.org/0000-0002-7496-7228

${ }^{2}$ Laboratório de Pesquisa em Fisioterapia Pulmonar (LFIP), Universidade Estadual de Londrina (UEL), Londrina, Paraná, Brasil. NAH - https://orcid.org/000-0002-5219-851X

${ }^{3}$ Departamento de Fisioterapia, Universidade Federal do Ceará (UFC), Fortaleza, Ceará, Brasil. Programa de Pós-Graduação em Fisioterapia e Funcionalidade, Universidade Federal do Ceará (UFC), Fortaleza, Ceará, Brasil. Programa de Pós-Graduação em Ciências Cardiovasculares, Universidade Federal do Ceará (UFC), Fortaleza, Ceará, Brasil. RBM - https://orcid.org/0000-0002$\underline{8048-3393}$
} 


\section{Abstract}

The current document provides guidelines for physiotherapy assistance to patients with suspected or diagnosed COVID-19, focusing on physiotherapy interventions that should be used with caution or even avoided. Amidst the COVID-19 pandemic, reducing the virus spread is a major concern for health agencies and healthcare professionals. Thus, recommendations related to the risk for generating aerosols, the risk of causing excessive energy expenditure and the risk of causing lung injury were provided. It is recommended to use with caution or avoid the use of Venturi mask, face tent oxygen mask, high flow nasal cannula, non-invasive ventilation with single circuit, hyperinflation bag system, moderate-to-high intensity physical exercises, as well as conventional mechanical ventilation settings. The physiotherapist who assists patients with COVID-19 must observe these guidelines to ensure an effective and safe care for both himself/herself and the patient.

Keywords: Physiotherapy; Standard of Care; COVID-19.

\section{Objetivo}

O objetivo do presente posicionamento é fornecer direcionamentos para o atendimento de Fisioterapia a pacientes com COVID-19, com foco nos recursos e técnicas que devem ser utilizados com cautela ou mesmo evitados, dependendo do quadro clínico do paciente e de fatores relacionados ao ambiente no qual o paciente se encontra.

\section{Contextualização}

A escolha dos recursos e técnicas fisioterapêuticas deve ser realizada de acordo com as indicações e contraindicações de cada uma delas, além de considerar os riscos e a individualidade do paciente. Em meio à pandemia da COVID-19, a redução da disseminação do vírus é uma preocupação importante dos órgãos e profissionais de saúde ${ }^{1,2}$. Deste modo, o fisioterapeuta que atende pacientes com COVID-19 precisa ser criterioso ao escolher recursos e técnicas que promovam os melhores benefícios da intervenção e, ao mesmo tempo, permitam que o profissional não se torne um agente transmissor do vírus ou se contamine ${ }^{3,4}$.

É importante lembrar que a COVID-19 pode apresentar diferentes quadros clínicos, atingindo a população de formas variadas, $\mathrm{como}^{1,5}$ :

- Doença leve: pacientes com infecção viral do trato respiratório superior sem complicações relevantes, que podem apresentar sintomas inespecíficos como febre, fadiga, tosse (produtiva ou improdutiva), anorexia, mal-estar, dores musculares, dor de garganta, dispneia, congestão nasal ou dor de cabeça. Com exceção das crianças, raramente, os pacientes com quadro leve apresentam diarreia, náusea e vômito. Vale ressaltar que idosos e indivíduos imunossuprimidos podem apresentar sintomas atípicos.

- Pneumonia: pacientes que apresentam febre e/ou sintomas no trato respiratório inferior etc.; com pneumonia detectada em exame de imagem, mas sem necessidade de oxigênio suplementar.

- Pneumonia grave: pacientes que apresentam febre ou sintomas no trato respiratório inferior associado a pelo menos um dos seguintes critérios: frequência respiratória (FR) > 30 respirações/min para adultos e FR > 40 para crianças; desconforto respiratório importante; saturação de pulso de oxigênio $\left(\mathrm{SpO}_{2}\right) \leq 93 \%$ em ar ambiente. 
- Síndrome do desconforto respiratório agudo (SDRA): pacientes que apresentam maior comprometimento respiratório, caracterizado por falência ventilatória com necessidade de ventilação mecânica. A SDRA pode ser classificada como leve, moderada ou grave, de acordo com o nível de oxigenação do paciente, por meio da razão $\mathrm{PaO}_{2} / \mathrm{FiO}_{2}^{1,5}$.

Embora o quadro clínico do paciente possa ser um fator determinante na escolha dos recursos e técnicas fisioterapêuticas, independentemente da classificação, deve-se considerar que existe um grande risco de disseminação do vírus e, portanto, o planejamento fisioterápico deverá ser adaptado ou adequado de acordo com os fatores ambientais disponíveis.

Além disso, infelizmente, nem todos os hospitais do Brasil têm recursos, tecnologia, estrutura física, ou mesmo equipamentos de proteção individual (EPI) ideais para serem utilizados frente ao novo coronavírus 6 . Portanto, é necessário considerar que vários hospitais brasileiros podem não contar, por exemplo, com número suficiente de: unidades de isolamento respiratório com pressão negativa; equipamentos de ventilação não-invasiva com circuito duplo, filtro High Efficiency Particulate Arrestance (HEPA) para o ramo expiratório do ventilador mecânico, máscaras de ventilação não invasiva (VNI) totalmente vedadas à face, película protetora para evitar lesão de pele, e ajuste da interface com o mínimo de vazamento para o ambiente; entre outros recursos que visam a prevenção e o controle da doença ${ }^{5-7}$.

A atuação do fisioterapeuta respiratório e do fisioterapeuta em terapia intensiva no tratamento de pacientes com infecções respiratórias já está bem descrita na literatura ${ }^{8-11}$. Contudo, também tem sido demonstrado que alguns recursos e técnicas utilizados pelo fisioterapeuta apresentam grande potencial para a disseminação de gotículas e aerossóis, o que pode aumentar a transmissão dos microrganismos causadores das infecções respiratórias como o SARS-CoV-2. Além disso, a utilização de algumas técnicas fisioterapêuticas pode causar gasto energético excessivo ou até aumentar o risco de injúria pulmonar. Todos esses pontos devem ser levados em consideração pelo fisioterapeuta ao atender o paciente com COVID-19.

Evidências científicas sugerem que o uso de VNI e de hiperinsuflação com ressuscitador manual antes da intubação podem aumentar o risco de transmissão de infecções respiratórias ${ }^{12}$. Sabe-se também que o uso de VNI e a aplicação de Fisioterapia Respiratória em pacientes com condições respiratórias foi capaz de gerar gotículas de grande tamanho $(>10 \mu \mathrm{m})$, as quais foram lançadas a até 1 metro de distância ${ }^{13}$. Além disso, observou-se que o SARS-CoV-2 permaneceu estável em superfícies como plástico e metal por até 72 horas $^{14}$. Isso nos faz refletir sobre os cuidados com equipamentos comumente utilizados no atendimento a pacientes com infecções respiratórias como o estetoscópio, além dos cuidados com o manuseio de pacientes com COVID-19. É importante analisar cuidadosamente a necessidade de cada técnica, uma vez que existem relatos também de que os pacientes geralmente não apresentam secreções pulmonares em abundância ${ }^{15}$.

Outra preocupação em relação às técnicas utilizadas pelo fisioterapeuta está relacionada ao aumento excessivo do gasto energético, como a mobilização ativa ou até o exercício físico ${ }^{16,17}$, e está voltada principalmente a pacientes críticos, ou seja, aqueles que desenvolvem SDRA. A hipótese é de que o gasto energético excessivo possa sobrecarregar os músculos respiratórios e precipitar um quadro de falência respiratória. Contudo, na maioria das vezes os pacientes com SDRA necessitam ser intubados e submetidos à ventilação mecânica invasiva ${ }^{1,7}$, e muitas vezes são sedados ou até curarizados, o que reduziria o gasto energético. Pacientes com COVID-19 frequentemente apresentam febre, que é um achado intrinsicamente ligado a um maior gasto energético e que não recomenda a prática de mobilização precoce e exercício físico ${ }^{18}$. 
Durante o atendimento a pacientes com COVID-19, o fisioterapeuta deve estar atento também quanto ao manejo da ventilação mecânica invasiva, principalmente nos pacientes que desenvolveram SDRA. Diversos estudos já demonstraram que o uso de parâmetros como volume e pressão mais elevados podem causar injúria pulmonar e estão associados à maior mortalidade ${ }^{19-21}$. Dessa forma, pacientes com SDRA devem ser submetidos a uma estratégia ventilatória protetora, a qual tem sido sugerida também para pacientes com SDRA em decorrência da COVID-19 ${ }^{1,7}$.

Com base no exposto acima, documentos nacionais e internacionais têm recomendado cautela ou até sugerido evitar alguns recursos e técnicas utilizadas pelo fisioterapeuta no manejo de pacientes com COVID-19. Essa cautela é reforçada por relatos de que um número considerável de profissionais de saúde tem se infectado com o SARS-CoV-2 em diversos países.

Direcionamentos mais específicos a respeito dos tópicos previamente abordados serão apresentados a seguir. Para fins didáticos, os diferentes recursos e técnicas fisioterápicas que costumam ser utilizados no atendimento a pacientes com disfunção respiratória semelhante à apresentada por pacientes com COVID-19 foram categorizados de modo que a recomendação de cautela no emprego fosse claramente identificada, como sendo referente:

- à geração de gotículas e aerossóis;

- ao gasto energético excessivo; $\mathrm{e}$

- ao risco de injúria pulmonar.

\section{Recursos e técnicas que devem ser utilizados com cautela devido à geração de gotículas e aerossóis:}

- Oxigenoterapia: para a promoção da $\mathrm{SpO}_{2}$ alvo, recomenda-se evitar ou utilizar com cautela o uso das seguintes interfaces: máscara de Venturi, tenda de oxigênio e cânula nasal de alto $\underline{\text { fluxo }}{ }^{7}$. Além disso, sugere-se evitar o uso de oxigênio em excesso $\left(\mathrm{SpO}_{2}\right.$ acima de $\left.96 \%\right)$ devido ao risco de lesão pulmonar induzida pela toxicidade do oxigênio, principalmente em pacientes com SDRA 4 .

Nota: Recomendações internacionais sugerem o uso da cânula nasal de alto fluxo para pacientes com hipoxemia leve à moderada sem evolução de piora, sendo um recurso para ser utilizado antes da necessidade de assistência ventilatória mecânical ${ }^{6}$; contudo, o uso de cânula nasal de alto fluxo deve ser restrito quando houver condições controladas de segurança ambiental (p. ex., quarto com isolamento aéreo e pressão negativa), sendo que o uso de fluxo menor que $30 \mathrm{~L} /$ min pode reduzir o potencial de transmissão viral ${ }^{4}$.

- VNI: a ASSOBRAFIR publicou recentemente um posicionamento sobre o assunto ${ }^{22}$ que está de acordo com um posicionamento internacional previamente publicado, ou seja, recomenda-se não utilizar VNI de forma rotineira ${ }^{4}$. Além disso, não se recomenda utilizar como recurso de resgate em novo quadro de piora da insuficiência respiratória aguda. Recomenda-se fortemente não utilizar VNI na ausência de condições controladas de segurança ambiental, ou seja, uso de interface completamente vedada à face, uso de circuito duplo e filtro HEPA no ramo expiratório, e sala com pressão negativa ${ }^{5,7,15,22}$. Na necessidade da utilização de VNI com circuito único, recomenda-se a utilização de circuito com orifício de exalação no próprio circuito (e não na máscara), e filtro HEPA entre a máscara e o orifício de exalação $0^{23}$.

Nota: segundo a experiência da equipe do Primeiro Hospital Afiliado, Universidade de 
Zhejiang (China), a VNI não dever ser utilizada nos pacientes com COVID-19 que falharam em reverter o quadro de insuficiência respiratória aguda com cânula nasal de alto fluxo ${ }^{5}$.

- Nebulização: evitar o uso de copo de nebulização para fins de umidificação e fluidificação de secreção pulmonar; evitar inalação convencional com fluxo de oxigênio ou de ar comprimido ${ }^{7}$. Sugere-se o uso de inaladores dosimetrados e espaçadores para a administração de broncodilatadores?

- Técnicas ou recursos para remoção de secreção brônquica: qualquer técnica que possa provocar tosse, como percussão, vibração e compressão torácicas, tosse manualmente assistida, oscilação oral de alta frequência (Shaker ${ }^{\circledR}$, Flutter $\left.{ }^{\circledR}\right)$, oscilação de alta frequência da parede

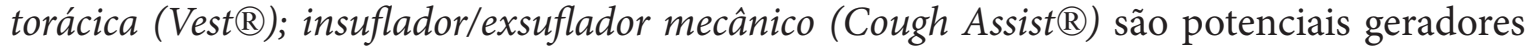
de gotículas/aerossóis e devem ser utilizadas apenas quando houver plena indicação e necessidade e com o devido uso de EPI em pacientes com COVID-1915,17,24. Achados prévios na literatura têm demonstrado que a infecção cursa com tosse seca e não costuma causar consolidações exsudativas ${ }^{4,25}$.

Nota 1: instrumentos como osciladores de alta frequência da parede torácica e insuflador/ exsuflador mecânico devem ser utilizados apenas em pacientes que, previamente à COVID-19, necessitavam desse auxílio ${ }^{15,24}$.

Nota 2: aspiração endotraqueal de rotina deve ser evitada. Quando realizada, é recomendado que seja em sistema de aspiração fechado.

- Hiperinsuflação manual: evitar o uso do ressuscitador manual como recurso para aumento do volume pulmonar. Para pacientes em ventilação mecânica invasiva, sugere-se alternativamente o uso do próprio ventilador ${ }^{4}$.

Observação: Importante ressaltar a necessidade de equipamentos de proteção individual que protejam contra aerossóis (p. ex., máscara N95 e óculos de proteção) sempre que forem ser realizados procedimentos que possam gerar aerossóis ${ }^{4}$.

\section{Recursos e técnicas que devem ser utilizados com cautela devido ao gasto energético excessivo:}

- Exercícios físicos: deve-se evitar exercícios com intensidade acima de 3 METs ou que induzam à sensação de dispneia maior que 3 na escala de Borg (CR10), como cicloergometria com carga acima de $50 \mathrm{~W}$, caminhada acima de $4 \mathrm{Km} / \mathrm{h}$ em superfície plana etc. ${ }^{17}$.

Nota 1: sugere-se cautela ao realizar exercícios em pacientes com tempo de diagnóstico de COVID-19 $\leq 7$ dias e/ou tempo entre o início dos sintomas e o surgimento de dispneia leve $\leq 3$ dias, mesmo em pacientes com doença leve, pois esses pacientes apresentam chance de evolução rápida para estados mais graves ${ }^{17}$. Os pacientes devem ser encorajados a manteremse apenas funcionais, realizando exercícios simples ou de atividade de vida diária, evitando gastar muito tempo do dia em atividades e posturas sedentárias ${ }^{4,26}$.

Nota 2: são contraindicações a realização de exercício físico de qualquer intensidade: febre, instabilidade hemodinâmica, progressão de imagem pulmonar em 24 a $48 \mathrm{~h}$ maior que 50\%, pressão intracraniana elevada, falta de cooperação ${ }^{17}$.

- Outras estratégias: assim como para o exercício físico, técnicas como treinamento muscular respiratório e espirometria de incentivo, que podem demandar maior gasto energético, caso sejam utilizadas, devem ter adequado controle de intensidade. 


\section{Recursos e técnicas que devem ser utilizados com cautela ou evitadas devido ao risco de injúria pulmonar:}

- Hiperinsuflação manual: o uso do ressuscitador manual não é recomendado. Além da geração de aerossóis e do risco de contaminação associado ao uso em pacientes em ventilação espontânea, causam desconexão e despressurização das vias aéreas quando utilizado em pacientes sob ventilação mecânica, predispondo à injúria pulmonar $7^{15,24}$.

- Estratégia convencional de ventilação mecânica invasiva: deve-se evitar estratégias convencionais e dar preferência às estratégias protetoras. Detalhes sobre este tópico poderão ser encontrados na comunicação oficial da ASSOBRAFIR ${ }^{22}$.

\section{Considerações Finais}

Considerando que esforços para minimizar a disseminação viral devem partir de todos os membros da equipe, a assistência fisioterapêutica deve estar devidamente pautada na avaliação e reavaliação dos pacientes com COVID-19, sendo observadas indicações e contraindicações, bem como os riscos tanto para o paciente quanto para a equipe, garantindo qualidade da assistência prestada.

Pacientes frágeis e/ou com morbidades prévias, em especial aqueles com doença cardíaca ou pulmonar crônica, diabetes ou hipertensão, são mais propensos à necessidade de intervenção pela Fisioterapia Respiratória; portanto, devem ser avaliados e constantemente monitorizados.

Poucas evidências estão disponíveis até o momento sobre quais recursos e técnicas fisioterapêuticas que devem ser utilizadas com cautela ou evitadas no atendimento da Fisioterapia a pacientes com COVID-19; logo, as recomendações aqui apresentadas se baseiam principalmente na opinião de especialistas e nos direcionamentos fornecidos por algumas entidades internacionais.

\section{Referências}

1. WHO/OMS. Clinical management of severe acute respiratory infection (SARI) when COVID-19 disease is suspected: Interim guidance, 13 March 2020 [Internet]. Geneva: World Health Organization; 2020 [cited 2020 Mar 22]. Available from: https://www.who.int/docs/default-source/coronaviruse/ clinical-management-of-novel-cov.pdf.

2. Brasil. Ministério da Saúde. Coronavírus: profissionais e gestores de saúde [Internet]. Brasília: Ministério da Saúde; 2020 [cited 2020 Mar 24]. Available from: https://coronavirus.saude.gov.br/.

3. WHO. Surface sampling of coronavirus disease (COVID-19): a practical "how to" protocol for health care and public health professionals [Internet]. Geneva: World Health Organization; 2020 [cited 2020 Mar 23]. Available from: https://apps.who.int/iris/handle/10665/331058.

4. Thomas P, Baldwin C, Bissett B, Boden I, Gosselink R, Granger CL, et al. Physiotherapy management for COVID-19 in the acute hospital setting: clinical practice recommendations. J Physiother. 2020 Apr;66(2):73-82. doi: 10.1016/j.jphys.2020.03.011. Epub 2020 Mar 30.

5. Handbook of COVID-19 Prevention and Treatment [Internet]. London: ALNAP; 2020 Mar 18 [cited 2020 Mar 23]. Available from: https://www.alnap.org/help-library/handbook-of-covid-19prevention-and-treatment. 
6. ANVISA. Nota técnica GVIMS/GGTES/ANVISA nº 04/2020. Orientações para serviços de saúde: Medidas de prevenção e controle que devem ser adotadas durante a assistência aos casos suspeitos ou confirmados de infecção pelo novo Coronavírus (SARS-COV-2) [Internet]. Brasília: Agência Nacional de Vigilância Sanitária; 2020 [cited 2020 Mar 23]. Available from: https://www.amib.org.br/fileadmin/user upload/amib/2020/marco/21/Nota Tecnica n 04-2020 GVIMS-GGTES-ANVISA-ATUALIZADA.pdf

7. AMIB. Orientações sobre o manuseio do paciente com pneumonia e insuficiência respiratória devido a infecção pelo Coronavírus (SARS-CoV-2) [Internet]. São Paulo: Associação de Medicina Intensiva Brasileira; 2020 [cited 2020 Mar 22]. Available from: http://www.amib.org.br/fileadmin/user upload/amib/2020/marco/20/1 Orientacoes sobre o manuseio do paciente com pneumonia e insuficiencia respiratoria devido a infeccao pelo Coronavirus ai.pdf.

8. Strickland SL, Rubin BK, Drescher GS, Haas CF, O’Malley CA, Volsko TA, et al. AARC clinical practice guideline: effectiveness of nonpharmacologic airway clearance therapies in hospitalized patients. Respir Care. 2013 Dec;58(12):2187-93. doi: 10.4187/respcare.02925.

9. Yang M, Yan Y, Yin X, Wang BY, Wu T, Liu GJ, et al. Chest physiotherapy for pneumonia in adults. Cochrane Database Syst Rev. 2013 Feb 28;(2):CD006338. doi: 10.1002/14651858.CD006338.pub3.

10. Larsen T, Lee A, Brooks D, Michieli S, Robson M, Veens J, et al. Effect of Early Mobility as a Physiotherapy Treatment for Pneumonia: A Systematic Review and Meta-Analysis. Physiother Can. Winter 2019;71(1):82-89. doi: 10.3138/ptc.2017-51.ep

11. Bjorkqvist M, Wiberg B, Bodin L, Barany M, Holmberg H. Bottle-blowing in hospitaltreated patients with community-acquired pneumonia. Scand J Infect Dis. 1997;29(1):77-82. doi: $10.3109 / 00365549709008669$.

12. Tran K, Cimon K, Severn M, Pessoa-Silva CL, Conly J. Aerosol generating procedures and risk of transmission of acute respiratory infections to healthcare workers: a systematic review. PLoS One. 2012;7(4):e35797. doi: 10.1371/journal.pone.0035797. Epub 2012 Apr 26.

13. Simonds AK, Hanak A, Chatwin M, Morrell M, Hall A, Parker KH, et al. Evaluation of droplet dispersion during non-invasive ventilation, oxygen therapy, nebuliser treatment and chest physiotherapy in clinical practice: implications for management of pandemic influenza and other airborne infections. Health Technol Assess. 2010 Oct;14(46):131-172. doi: 10.3310/hta14460-02.

14. van Doremalen N, Bushmaker T, Morris DH, Holbrook MG, Gamble A, Williamson BN, et al. Aerosol and Surface Stability of SARS-CoV-2 as Compared with SARS-CoV-1. N Engl J Med. 2020 Apr 16;382(16):1564-1567. doi: 10.1056/NEJMc2004973. Epub 2020 Mar 17.

15. Moses R. COVID 19: Respiratory Physiotherapy On Call Information and Guidance. Version 2 dated 14th March 2020 [Internet]. London: Chartered Society of Physiotherapy; 2020 [cited 2020 Mar 22]. Available from: https://www.csp.org.uk/system/files/documents/2020-03/COVID\%20 19\%20Respiratory\%20Physiotherapy\%200n\%20Call\%20Information\%20and\%20Guidance $\% 20 \mathrm{~V} 2$. pdf. https://www.csp.org.uk/documents/coronavirus-respiratory-physiotherapy-call-guidance

16. Indicazione per la Fisioterapia respiratoria in pazienti con infezione da COVID-19, aggiornato al 16/03/2020 [Internet]. Roma: Associazione Riabilitatori dell'insufficienza Respiratoria; 2020 [cited 2020 Mar 23]. Available from: https://aifi.net/emergenza-covid-19-indicazioni-per-fisioterapisti-epazienti/ 
17. Grupo de Interesse em Fisioterapia Cardiorrespiratória. Fisioterapia respiratória em pessoas com COVID-19 [Internet]. [São Domingos de Rana]: Associação Portuguesa de Fisioterapeutas; 2020 May 4 [cited 2010 Mar 23]. Available from: https://www.gifcr-apf.com/

18. Gosselink R, Bott J, Johnson M, Dean E, Nava S, Norrenberg M, et al. Physiotherapy for adult patients with critical illness: recommendations of the European Respiratory Society and European Society of Intensive Care Medicine Task Force on Physiotherapy for Critically Ill Patients. Intensive Care Med. 2008 Jul;34(7):1188-99. doi: 10.1007/s00134-008-1026-7. Epub 2008 Feb 19.

19. Amato MB, Barbas CS, Medeiros DM, Magaldi RB, Schettino GP, Lorenzi-Filho G, et al. Effect of a protective-ventilation strategy on mortality in the acute respiratory distress syndrome. N Engl J Med. 1998 Feb 5;338(6):347-54. doi: 10.1056/NEJM199802053380602.

20. Amato MB, Meade MO, Slutsky AS, Brochard L,Costa EL, Schoenfeld DA, et al. Driving pressure and survival in the acute respiratory distress syndrome. N Engl J Med. 2015 Jan 31;372(8):747-755. doi: 10.1056/nejmsa1410639

21. Guerin C, Papazian L, Reignier J, Ayzac L, Loundou A, Forel J-M. Effect of driving pressure on mortality in ARDS patients during lung protective mechanical ventilation in two randomized controlled trials. Crit Care. 2016 Nov 29;20(1):384. doi: 10.1186/s13054-016-1556-2.

22. Martinez BP, Andrade FMD, Roncalli A, Martins JA, Cavalheiro LV, Matte DL, et al. Intervenção na Insuficiência Respiratória Aguda. Comunicação Oficial COVID-19 ASSOBRAFIR [Internet]. São Paulo: ASSOBRAFIR, 2020 [cited 2020 Mar 23]. Available from: https://assobrafir.com.br/covid-19 vni/.

23. British Thoracic Society. COVID-19: information for the respiratory Community [Internet]. London: British Thoracic Society; 2020 [cited 2020 Mar 24]. Available from: https://www.britthoracic.org.uk/about-us/covid-19-information-for-the-respiratory-community/.

24. Physioplus. Respiratory Management of COVID 19. [cited 2020 Mar 22]. Available from: https:// members.physio-pedia.com.

25. Han R, Huang L, Jiang H, Dong J, Peng H, Zhang D. Early Clinical and CT Manifestations of Coronavirus Disease 2019 (COVID-19) Pneumonia. AJR Am J Roentgenol. 2020 Aug;215(2):338343. doi: 10.2214/AJR.20.22961. Epub 2020 Mar 17.

26. Arbillaga A, Pardàs M, Escudero R, Rodríguez R, Alcaraz V, Llanes S, et al. Fisioterapia respiratoria en el manejo del paciente con covid-19: recomendaciones generales. Versión 1 [Internet]. Barcelona: Sociedad Española de Neumología y Cirugía Torácica; 2020 Mar 26 [cited 2020 Apr 01]. Available from: http://svmefr.com/wp-content/uploads/2020/03/COVID19-SEPAR-26 03 20.pdf.

Submissão em: 07/06/2020

Aceito em: 15/07/2020 